\title{
Video-assisted minimally invasive approach in clinical bilateral lung transplantation
}

\author{
Stefan Fischer, MD, MSc, Martin Strüber, MD, Andre R. Simon, MD, Marcel Anssar, MD, Michaela Wilhelmi, MD, \\ Rainer G. Leyh, MD, Wolfgang Harringer, MD, PhD, and Axel Haverich, MD, PhD, Hannover, Germany
}

From Hannover Lung Transplant Program, Division of Thoracic and Cardiovascular Surgery, Hannover Medical School, Hannover, Germany.

Received for publication April 4, 2001; accepted for publication June 22, 2001.

Address for reprints: Martin Strüber, MD, Hannover Lung Transplant Program, Division of Thoracic and Cardiovascular Surgery, Hannover Medical School, Carl-NeubergStrasse 1, 30625 Hannover, Germany (E-mail: strueber@thg.mh-hannover.de).

J Thorac Cardiovasc Surg 2001;122:1196-8

Copyright (C) 2001 by The American Association for Thoracic Surgery

$0022-5223 / 2001 \$ 35.00+0 \quad \mathbf{1 2 / 5 4 / 1 1 8 2 7 2}$

doi:10.1067/mtc.2001.118272

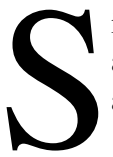

ince the report on the first successful clinical lung transplant procedure by Cooper and the Toronto Lung Transplant Group ${ }^{1}$ in 1986, the operation has become an accepted treatment modality for end-stage pulmonary failure in various diseases.

The approach to bilateral lung transplantation has changed significantly since the inception of the operation. The original double-lung technique was performed through a median sternotomy with the recipient supported by cardiopulmonary bypass. The donor lungs were implanted en bloc with a single tracheal anastomosis. Although this operation was successful, its limitations became apparent as it was extended to more difficult cases. In 1989 the bilateral sequential operation was introduced and has become the current standard approach. The lungs are sequentially and separately implanted through an anterior transverse thoracosternotomy (clamshell incision). ${ }^{2}$ This incision provides excellent exposure of the pleural space. However, many disadvantageous effects are associated with this extremely invasive approach. According to the literature and our own clinical observations, early postoperative pain after thoracotomy is a significant problem. Additionally, chronic post-thoracotomy neuralgia is seen in many patients, which affects the quality of life and often requires long-term use of analgesic drugs. ${ }^{3}$ For the transplanted lung to function optimally, sufficient breathing activity is critical. Postoperative pain, however, leads to flat chest movements and insufficient graft ventilation, which further increase the likelihood of pneumonia. Currently, most immunosuppressive regimens include the application of steroids, which are widely known to impair wound healing. This is especially true in large wounds such as the clamshell incision. ${ }^{4}$ Wound infections including osteomyelitis after sternotomy are frequently described complications after surgical intervention, especially in the immunosuppressed patient, and often necessitate reoperation. In single cases these complications have caused mediastinitis and sepsis with lethal outcomes.

At the Hanover Lung Transplant Program, 50 to 60 lung transplants are performed throughout the year. The majority of our patients are either young female recipients or elderly patients receiving long-term corticoid therapy. Whereas the latter group of patients will greatly benefit from a less-invasive approach without sternotomy because of impaired steroid-induced wound healing, an improved cosmetic outcome is likely important to the majority of patients undergoing lung transplantation. We have developed a novel minimally invasive video-assisted approach, which we now routinely use in all patients undergoing bilateral lung transplantation.

\section{Technique}

The patient is routinely intubated with a double-lumen intratracheal tube and placed on the operating table in the supine position. The skin incisions $(10-12 \mathrm{~cm})$ are performed above the fifth intercostal space. In female patients the incisions are placed under the breast to ensure the best possible cosmetic results. The chest is entered bilaterally through a 12- to $15-\mathrm{cm}$ anterolateral thoracotomy via the fifth intercostal space. Ligation of the right and left internal thoracic arteries is avoided. For hilar dissection the corresponding lung is deflated, the phrenic nerve is identified, and the first branches of the pulmonary artery and vein are snared (Figure 1). So that vision can be improved, a camera with a light source, normally used for thoracoscopic interventions, is placed in the chest through a 1$\mathrm{cm}$ incision in the lower and lateral part of the chest. This incision is ultimately used for postoperative chest drainage (Figure 1). Thus, the operating field can be illuminated perfectly, focusing on the site of dissection. In addition, the dissection maneuver can be viewed on a screen by the assistant and operating room nurse, ensuring easy coordination 

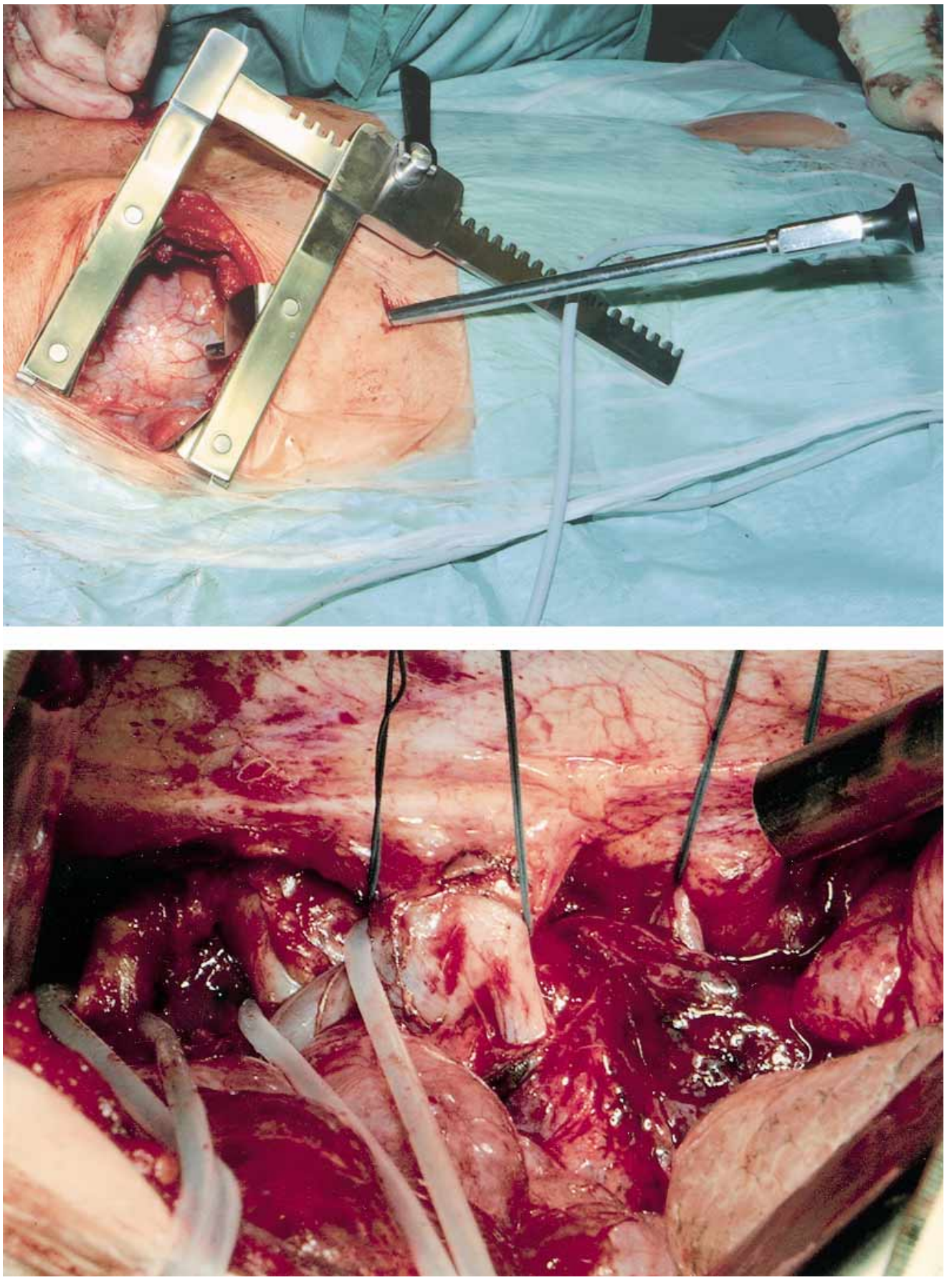

Figure 1. Upper panel: A camera, usually used for thoracoscopic interventions, is placed into the chest through a 1-cm incision to illuminate the site of dissection. At the end of the operation this incision is used for chest drainage. Lower panel: The phrenic nerve is identified and the hilar structures are snared. In the right upper corner the tip of the camera appears.

of the operation. The anastomoses of the bronchus, pulmonary vein, and artery are performed according to generally accepted techniques, including a running suture line for the bronchial anastomosis. ${ }^{5}$ The incisions are closed by 2 to 3 absorbable pericostal stitches, the subcutaneous tissue layers are adapted anatomically by means of an absorbable running suture, and the skin is intracutaneously closed with a monophilic running suture. Our patients have less pain with a smoother convalescence and a more satisfactory cosmetic result than do patients operated on with the standard technique (Figure 2). 


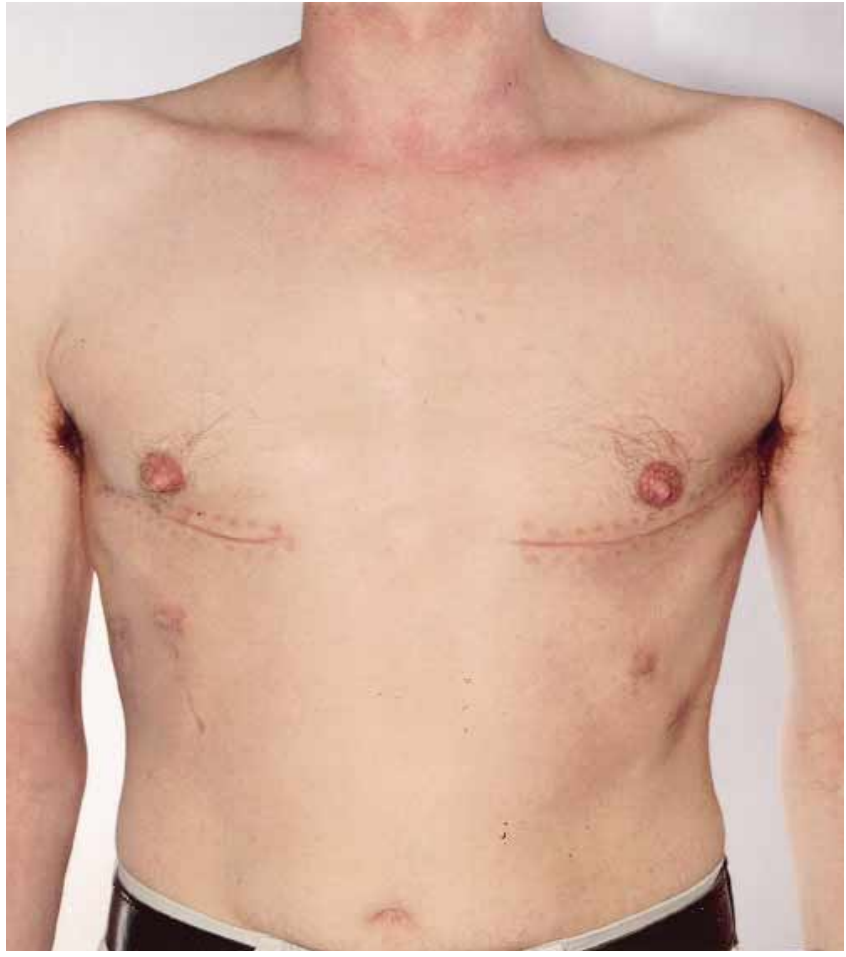

Figure 2. Cosmetic outcome after minimally invasive bilateral lung transplantation.

Five of our recipients who underwent minimally invasive bilateral lung transplantation became hemodynamically unstable during the operation and required cardiopulmonary bypass. For atrial cannulation we use a flattened $10-\mathrm{mm}$ venous cannula (Medtronic DLP, Grand Rapids, Mich) and a 7-mm aortic cannula (Jostra Medizintechnik AG, Hirrlingen, Germany), shown in Figure 3. We routinely use both for minimally invasive repair of atrial septal defects. So far we have experienced no difficulties in cannulating the ascending aorta and right atrium or in continuing the transplant procedure through the small incision after the cannulas were inserted.

Limitations of this minimally invasive approach may be obesity and a very small thoracic cavity resulting from elevation of the diaphragm in patients with end-stage pulmonary fibrosis.

\section{Conclusion}

The extremely invasive clamshell incision, which is usually used in bilateral lung transplantation, provides many clinically relevant

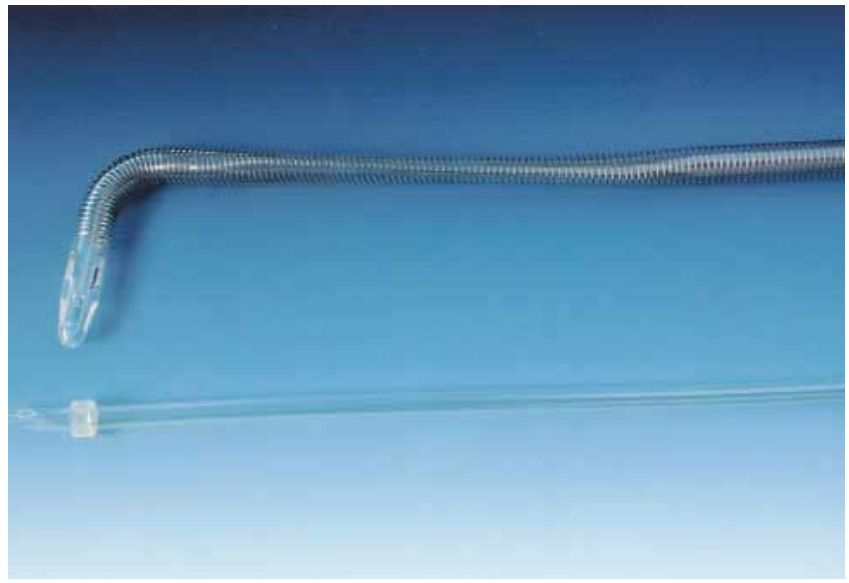

Figure 3. Cannulas for atrial and aortic cannulation when cardiopulmonary bypass support is required. The upper $10-\mathrm{mm}$ venous cannula is flattened and the lower arterial cannula is only $7 \mathrm{~mm}$ in diameter. These cannulas allow for cannulation through the small incisions without conversion to the conventional clamshell incision.

problems and complications during the early and late postoperative periods. Our novel video-assisted minimally invasive approach leads to lessened postoperative pain, improved convalescence, and satisfactory cosmetic results. Conversion to the conventional approach may be necessary in individual cases such as in patients having retransplantation. On the basis of our experience, we believe this novel approach to be safe and more beneficial for the patient than the traditional anterior transverse thoracosternotomy. Thus, at our program we use this strategy in patients undergoing bilateral lung transplantation without exception.

\section{References}

1. Toronto Lung Transplant Group. Unilateral lung transplantation for pulmonary fibrosis. $N$ Engl J Med. 1986;314:1140-5.

2. Patterson GA. Indications: unilateral, bilateral, heart-lung, and lobar transplant procedures. Clin Chest Med. 1997;18:225-30.

3. Rogers ML, Duffy JP. Surgical aspects of chronic post-thoracotomy pain. Eur J Cardiothorac Surg. 2000;18:711-6.

4. Meyers BF, Sundaresan RS, Guthrie T, Cooper JD, Patterson GA. Bilateral sequential lung transplantation without sternal division eliminates posttransplantation sternal complications. J Thorac Cardiovasc Surg. 1999;117:358-64.

5. Meyers BF, Lynch J, Trulock EP, Guthrie TJ, Cooper JD, Patterson GA. Lung transplantation: a decade of experience. Ann Surg. 1999; 230:362-70; discussion 70-1. 